\title{
Estudio analítico sobre plataformas multimedia de memoria cultural, apoyada en heurísticas de sociabilidad y usabilidad
}

\author{
Juliana Campos Lobo
}

Recibido: 30 de marzo de 2015

Aceptado: 21 de mayo de 2015

\section{Resumen}

Se pretende hacer una evaluación analítica de las heurísticas de usabilidad y sociabilidad aplicándolas a la contribución y a la colaboración de plataformas digitales multimedia las cuales están relacionadas con la memoria cultural, como puede ser Museo da Pessoa, Portal Porta Curtas y Proyecto Cidade Velha. Este análisis es financiado por las heurísticas de usabilidad y sociabilidad, desarrolladas por Preece y Shneiderman (2009). Como resultados, se señalan las limitaciones y el potencial de las plataformas refiriéndose a las propias heurísticas, basado en las evaluaciones e investigaciones de los especialistas del doctorado en el área de información y comunicación de plataformas digitales.

\section{Pallabras clave}

Plataformas multimedia, Memoria Cultural, Usabilidad, Sociabilidad, Colaboración

\section{Analytical study on multimedia platforms of cultural memory, supported on heuristics of usability and sociability}

\section{Abstract}

The aim of this paper is to an analytical evaluation of the heuristics of usability and sociability applied to the contribution and cooperation in the context of multimedia digital platforms related to cultural memory, including the Pessoa Museum, Portal Porta Curtas and Cidade Velha Project. The analysis is subsidized by the heuristics of usability and sociability, developed by Preece and Shneiderman (2009). As results, still are pointed constraints and potential of platforms that concern these heuristics, based on the evaluation of embedded experts doctoral research processes in the area of information and communication on digital platforms.

\section{Keywords}

Multimedia platforms; Cultural Memory; Usability; Sociability; Collaboration

http://dx.doi.org/10.5209/rev CDMU.2015.v26.50630 


\section{INTRODUCCIÓN}

Las transformaciones que ofrecen las tecnologías de la información y comunicación es el resultado del avance de internet, el cual permitió el cruce de barreras relacionadas con el espacio-tiempo además de crear nuevos procesos de mediación e interacción en la comunicación humana. Sin embargo, cabe destacar que, a pesar de que internet haya provocado una revolución mucho más amplia y profunda que los medios masivos de comunicación, ésta es la parte más visible y popular de la llamada Revolución Digital, la cual permitió la caracterización de la Sociedad de la Información o de la Sociedad en Red, acuñado por Manuel Castells (2001).

Fueron con los primeros usuarios de la Web World Wide, que conocieron los primeros cambios tecnológicos, sociales y culturales, cuando a través de un simple clic, era posible ir de un sitio a otro y acceder a una gran variedad de contenidos posibles. Años más tarde con la aparición de la Web 2.0, de simple usuarios pasamos a ser productores y colaboradores de información, ya que anteriormente la divulgación del contenido público, no estaba al alcance de un ciudadano común.

Por lo tanto, es esencialmente fundada por la democratización del uso de la red y por el open access (acceso directo) "que se define como la disponibilidad gratuita de la literatura en internet que permite que cualquier usuario pueda leer, descargar, copiar, imprimir, distribuir, buscar y enlazar información sin barreras financieras, legales o técnicas" (Budapest open acces initiative, 2002 ; Hilário, 2014), la sociedad en red permite un nuevo tipo de interacción comunicativa entre los agentes, en el cual, la colaboración se constituye como principal característica. En este escenario, la herramienta de la Web 2.0 trajo dinamismo, interactividad y flexibilidad a los contenidos y publicaciones, que en su defecto dejo de presentar una característica estética y proporcionó un espacio para la edición de contenidos no solo para los profesionales en esta área sino también para el propio usuario, facilitando así el uso de los solicitantes.

Con tantas ventajas es fundamental dar un vistazo a la potencialidad que ofrece la web, como la proyección de plataformas digitales multimedia, particularmente en este estudio, las plataformas de memoria cultural, documentan su contenido por medio de lo audiovisual, trayendo unas heurísticas de usabilidad y sociabilidad gracias a la colaboración del propio usuario. La intención de analizar este proceso es para poder dar al usuario un lugar privilegiado, en el cual se le da la posibilidad de construir y producir el propio contenido de la red.

\section{REVISIÓN BIBLIOGRÁFICA}

\section{La interacción hombre-ordenador y la plataforma digital}

A finales de la década de los 70 e inicio de los 80, fue con la creación de los primeros ordenadores personales de IBM, que se facilitó una experiencia entre design and user (diseño y usuario) el cual planteó una serie de cuestiones en relación con el acceso de los usuarios y la interfaz tecnológica (Chow et al., 2014: 256). A partir de ahí y para facilitar la interacción entre hombre-ordenador, la proyección tecnológica para el usuario se convierte en prioridad, ofreciendo así, las bases para la formación en el campo de estudio de la interacción entre el hombre y el ordenador $(\mathrm{HCl}$ o $\mathrm{IHC})$.

Según Preece et al. (1994), el área del $\mathrm{HCl}$ busca específicamente abordar los factores con respecto a la relación entre el ser humano y el ordenador: seguridad, sociabilidad, utilidad, eficiencia, eficacia y usabilidad. Su principio fundamental es la concepción de interfaces tecnológicas, en el que el usuario se convierte en la 
pieza fundamental para el desarrollo de sistemas intuitivos y amigables. Así al centrar la atención en el usuario, el área del $\mathrm{HCl}$ abarca otra práctica, que es conocida como Diseño Centrado en el Usuario (DCU o UCD) el cual contempla la creación de nuevas experiencias, atrayentes y eficientes para el usuario.

En esta interacción del ser humano-ordenador, se incluye también otros sistemas e interfaces tales como las plataformas digitales, que surgen como medio habitado/poblado en la web, que se utiliza principalmente para el lanzamiento de contenido, donde los usuarios pueden producirlos, compartirlos y distribuirlo, interactuando así con otros usuarios (West and Gallagher, 2004). En esta coyuntura, las redes sociales, sitios webs, blogs, entre otros, ganan un papel clave en el reto de poder facilitar al sujeto lo que busca, esto adquiere una postura diferente para estas plataformas, como puede ser la función de prosumer (productor + consumidor).

Sin embargo, diseñar estas plataformas no es tarea fácil, ya que estas deben alcanzar un número elevado de usuarios, que tengan diferentes habilidades y necesidades en el uso de los sistemas tecnológicos. Por eso, cuando se plantea algunas tendencias generales, es importante tener en cuenta el perfil del usuario y las características que adopta.

Esta evaluación analítica, observa los procesos de contribución y colaboración por parte del usuario, a través de las plataformas digitales multimedia de memoria cultural las cuales utilizan el recurso audiovisual como formato, para así preservar su contenido, como aspectos decisivos en la definición de estos últimos cambios.

\section{Contribución y colaboración en el ciberespacio}

Semánticamente, "el concepto de colaboración está relacionado con el concepto de contribución y cooperación, ya que envuelven un trabajo colectivo con un objetivo en común" (Barros, 1994, 38). Sin embargo, Preece y Shneiderman (2009) cuando tratan de los niveles de participación social de la mayoría de los Usuarios: reading, contributing, collaborating and leading (p. 16) colocan la contribución en una etapa/nivel anterior a la colaboración. Sin embargo, algunos de los autores hacen una observación ya que esta sucesión no es siempre perfecta o está completa, y que los usuarios puedan o no puedan progresar de una etapa para otra (Figura 1).

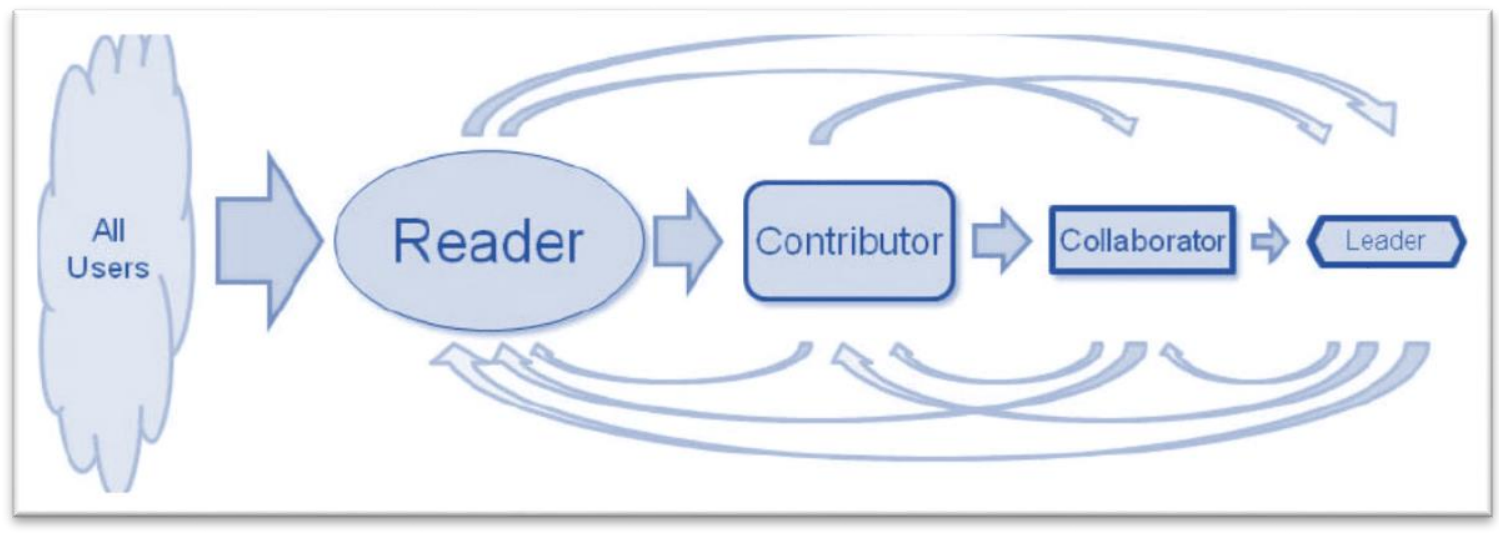


Figura 1: The Reader-to-Leader Framework (Preece e Shneiderman, 2009).

Para Denning y Yaholkovsky (2008), el concepto de colaboración abarca dos o más contribuyentes, colaborar y trabajar juntos para crear algo o compartir informaciones. Para Butler et al. (2008), cuando se trata de la participación en los medios de comunicación social, la contribución adoptara un papel individual e independiente, mientras que la colaboración presupone un trabajo en grupo. Sin embargo, las contribuciones individuales pueden traer beneficios substanciales para todos los participantes, aunque no sea directamente (Preece, 2000).

En el proceso de comunicación, con la introducción de los nuevos formatos y recursos de media, tanto la contribución como la colaboración se materializan a través de las plataformas multimedia, "creando nuevos espacios culturales, siendo capaz de cambiar las interacciones sociales y la estructura social en general" (Santaella; Noth, 2005: 11). Por eso, un factor esencial en este proceso, es el desarrollo de una base común, es decir, un entendimiento mutuo entre los sujetos, además de compartir las creencias religiosas y los parámetros (Convertino et al., 2008).

A partir de lo que es común, se vuelve más plausible la facilidad de comunicación y la eficiencia del acto de contribuir y colaborar, como, por ejemplo, en el caso del documental norte-americano Life in a Day, compuesto por imágenes, gravadas durante las 24 horas del día 24 de Julio de 2010, por personas comunes y de todas parte del mundo, en total, fueron 192 países que participaron, desde Australia hasta Zambia (Lobo, 2013).

Por lo tanto, en este estudio/análisis los niveles de contribución y colaboración por parte del Usuario, siguen la lógica de la sucesión de niveles de participación social estructurada por Preece y Shneiderman (2009), así como sus heurísticas de usabilidad y sociabilidad aplicadas a estos niveles. 


\section{La Memoria Cultural en el contexto de la mediación tecnológica}

Los procesos de comunicación poseen una relación concreta con la realidad y con la experiencia, la cual, según Duarte (1999), está vinculada a un conjunto de conocimientos basado en las costumbres, y por eso depende "de los mecanismos de la memoria, de la capacidad de recordar en el presente, el pasado y de prevenir el futuro, a partir del recuerdo presente del pasado" (Duarte, 1999: 5-6). A partir de ahí surgen dos procesos de señales opuesto: el recuerdo y la predicción.

El recuerdo es identificado por las marcas del pasado, que son evocados por los actos vividos en el presente, y que admiten el reconocimiento y la familiaridad (Duarte, 1999). La predicción, también se consolidó en el presente, con hechos que evocan el proceso de anticipación de efectos futuros en relación a esos hechos del presente. La memoria también se puede rescatar, por lo que existe en común con una comunidad concreta y que se extendió gracias a la convivencia directa/indirecta e inmediata, a esto se le denomina relaciones sociales.

De hecho, según el sociólogo francés Maurice Halbwachs (2006), la memoria individual existe a partir de una memoria colectiva (1) - término acuñado por él - donde los recuerdos se constituyen dentro de un grupo, es decir, a partir de las relaciones y de la interacción social. Además, el origen de algunas ideas, reflexiones, sentimientos, pasiones que se nos asignan, están inspirados en el grupo social que pertenecemos y al idioma que nos une.

Sin embargo, aunque la concepción de Halbwachs haya establecido un "territorio conceptual" para la memoria, la reutilización de estos estudios a través de las ciencias humanas y sociales trajo una separación de comprensión con respecto a la memoria individual (o neurobiológica) y la memoria colectiva (o socialhistorica). En la Psicología cognitiva, se reconoce tres principales "sistemas de memoria", los cuales son, la memoria sensorial, la memoria de trabajo (o memoria operacional y memoria de corta duración) y la memoria de larga duración, dicha memoria está a su vez dividida en memoria declarativa (que puede ser; memoria episódica o memoria semántica) y la memoria de procedimientos.

La cantidad de conceptos corresponde a la distinción de los objetos científicos. Sin embargo, si prestamos atención a Halbwachs (2006), la memoria individual existe a partir de una memoria colectiva, un vínculo entre las reminiscencias del sujeto y la del grupo social, en el cual este sujeto está incluido, puede referirse al idioma y al lazo cultural que reúne, lo cual consecuentemente genera una interacción social. Con esto, tanto el lazo cultural como la interacción social colaboran para constituir una memoria individual y colectiva, que juntas forman la memoria cultural.

¿Por qué memoria cultural? Primero decir que el hombre, como ser cultural (2) que es, no actúa solo, y por eso, su memoria cultural "está directamente unida al patrimonio de un pueblo, el cual a través de esta cultura genera, tomada en manifestaciones naturales, materiales, esto es un punto de referencia tanto en la identidad como en la fuente de inspiración" (Carneiro, 2006: 20). En otras palabras, son los elementos que forman la identidad cultural de un grupo, constituidos a lo largo de su historia. Por lo tanto, se refiere a sus antecedentes culturales, contribuyendo así a su forma de hacer, de sentir, de ser, de expresar, pues todo esto es un factor muy importante para la identificación del individuo en relación a si mismo y al grupo. 
Hay que destacar, que las tres plataformas analizadas poseen una característica en común; es decir, aquellos recursos tecnológicos que se relacionan con la memoria cultural, destacando que esta ya puede ser analizada en el contexto de mediación tecnológica. Uno de estos recursos mencionados está ligado a lo audiovisual, formato que es utilizado por el Museu da Pessoa, Portal Porta Curtas e Projecto Cidade Velha, para asi poder registrar datos de la memoria cultural, ya sea una declaración personal, un cortometraje o un documental histórico, respectivamente. Es decir, la memoria cultural puede ser documentada en nuevas áreas de interacción social y con la ayuda de las nuevas herramientas y recursos de preservación, las cuales contextualizaremos brevemente en el apartado 4.

\section{MÉTODO}

Para apoyar el propósito de este estudio, se utilizó un método de evaluación analítica, secundada por las heurísticas de usabilidad y sociabilidad, desarrolladas por Preece e Shneiderman (2009) y creada por especialistas (Lobo, Barbosa, Ferreira, Ramos, Santos, 2015) (3) vinculados a las investigaciones del doctorado y encuadrado en el marco de la información y comunicación en plataformas digitales.

La evaluación analítica, según Dix et al. (2004), se basa en las heurísticas, que corresponde a "guideline[s] or general principle[s] or rule[s] of thumb that can guide a design decision or be used to critique a decision that has already been made" (Dix et al., 2004: 324). Fue desarrollada por Jakob Nielsen y Rolf Molich como método para estructurar una crítica alrededor de un sistema que un utiliza un conjunto de heurísticas relativamente simple y generales.

El análisis se llevó a cabo en tres plataformas digitales de multimedia colaborativas, relacionadas a la memoria colectiva. Las cuales son: Museo da Pessoa, Portal Porta Curtas y Proyecto Cidade Velha.

\section{PROCEDIMIENTO}

\section{Caracterización de los expertos}

Para Dix et al. (2004), la idea general en torno a esta evaluación analítica con base heurísticas es que varios expertos pueden criticar, de forma independiente, un sistema para poder así detectar los problemas de usabilidad. La experiencia de Nielsen indica que entre tres y cinco especialistas es suficiente, pero que con cinco el resultado llega a tener un $75 \%$ de credibilidad para descubrir algunos problemas generales en la usabilidad.

Así que para garantir una coherencia en este estudio, serán cinco especialistas (Lobo, Barbosa, Ferreira, Ramos, Santos, 2015) vinculados en las investigaciones del doctorado, de información y comunicación en plataformas digitales que evaluaran las tres plataformas mencionadas en la sección anterior.

\section{Caracterización de las heurísticas}

Dada la variedad de plataformas con rasgos comunes, como puede ser los niveles/porcentajes de contribución y colaboración y además de la temática de lo audiovisual y de la memoria cultural, se presenta las heurísticas de usabilidad y sociabilidad (Tablas I y II) desarrolladas por Preece y Shneiderman (2009), que proporcionan una base para la evaluación analítica que aquí se propone. 
Usability

Low threshold interfaces for easily making

small contributions, e.g., no login
Sociability

Support for legitimate peripheral participation so that readers can gradually edge into contributing

High ceiling interfaces that allow large and frequent contributions

Visibility for users' contributions and frequency of views; aggregated over time

A change to build their reputation over time while performing satisfying tasks

Recognition for the highest quality and quantitu of contributions

Visibily of ratings and comments by community Recognition of a person's specific expertise members

Tools to undo vandalism, limit malicious users, Policies and norms for appropiate contributions control pornography and libel

Tabla I: Usability and sociability factors that may influence contributing (Preece e Shneiderman, 2009: 21).

\begin{tabular}{|c|c|}
\hline Usability & Sociability \\
\hline $\begin{array}{l}\text { Ways to locate relevant and competent } \\
\text { individuals to from collaborations }\end{array}$ & $\begin{array}{l}\text { An atmosphere to empathy and trust that } \\
\text { promotes belonging to the community and } \\
\text { willigness to work within groups to produce } \\
\text { something larger }\end{array}$ \\
\hline $\begin{array}{l}\text { Tools to collaborate: communicate within } \\
\text { groups, schedule projects, assign tasks, share } \\
\text { work products, request assistance }\end{array}$ & $\begin{array}{l}\text { Altruism: a desire to support the community, } \\
\text { desire to give back, willingness to reciprocate }\end{array}$ \\
\hline $\begin{array}{l}\text { Visible recognition and rewards for } \\
\text { collaborators e.g., authorship, citations, links, } \\
\text { acknowledgements }\end{array}$ & $\begin{array}{l}\text { The desire to develop a reputation for } \\
\text { themselves and their collaborators, their group } \\
\text { and community; the need to develop and } \\
\text { maintain one's status within the group }\end{array}$ \\
\hline $\begin{array}{l}\text { Ways to resolve differences (e.g., voting), } \\
\text { mediate disputes, and deal with unhelpful } \\
\text { collaborators }\end{array}$ & Respect for one's status within the community \\
\hline
\end{tabular}

Tabla II: Usability and sociability factors that may influence collaborating (Preece e Shneiderman, 2009: 23)

Un punto necesario a destacar, fue la elección de las heurísticas de usabilidad y sociabilidad, que además de ser imprescindibles para identificar la facilidad (Nielsen, 2012) para apoyar los objetivos, las influencias y las interacciones sociales que ocurren en las diferentes plataformas (Preece, 2001) son aspectos señalados por Preece e Shneiderman (2009) y están próximos a los niveles de contribución y colaboración del usuario.

\section{Caracterización de los objetos de estudio}

En el contexto histórico de las plataformas digitales de multimedia, se observa unas nuevas características estructurales y unos nuevos procesos constitutivos, los cuales han influenciado claramente, las formas expresivas de los últimos años. Para López-de-Quintana (2007), por ejemplo, "contrarrestar la ambigüedad del lenguaje y proporcionar una interfaz funcional al usuario final son imprescindibles herramientas que interpreten las estructuras lingüísticas en un contexto semántico". 
Se pueden observar estas nuevas características y procesos, en aquellas plataformas con herramientas tecnológicas aplicadas a la contribución y a la colaboración por parte del usuario. Como veremos a continuación, en este estudio, fueron exploradas analíticamente las plataformas; Museo da Pessoa, Portal Porta Curtas y Proyecto Cidade Velha, ya que estas reúnen las heurísticas de usabilidad y sociabilidad unidas a estos niveles.

\section{Museo da Pessoa}

La iniciativa por la que hoy en día se conoce el Museo da Pessoa nació a partir de una experiencia que se realizó en 1991, en el Museo de la Imagen y Sonido (Museo da Imagem e do Som (MIS) de San Paulo, durante la exposición de "Memória e Migração" (La Memoria y la Migración), la cual mostraba, la trayectoria de inmigrantes judíos que llegaban a Brasil, mostrando a través de innumerables actividades, las memorias de los judíos asentados en San Paulo. Dentro de la exposición, se habilitó un cuarto para aquellas personas que querían contar y registrar su historia de vida. Esta experiencia hizo que creciese una gran demanda, tanto por el espacio en si como por la propia historia, debido a la riqueza que revela cada historia contada. Gracias a esto, se concretizo la propia estructura del actual museo.

El Museo da Pessoa es considerado un museo virtual y colaborativo, el cual ofrece al visitante la oportunidad de formar parte de él, registrando su historia de vida a través del recurso audiovisual o asumiendo la función de curador, publicando sus propios vídeos, imagines e historias. Actualmente la base del museo cuenta con más de 16 mil testimonios, en vídeos, textos y audios sin contar las 60 mil fotografías y documentos digitalizados.

\section{Portall Porta Curtas}

Creado en agosto de 2002, la plataforma Portal Porta Curtas aparte de catalogar/seleccionar en internet los mejores cortometrajes brasileños también crea un panel representativo en la producción nacional de cortos, técnicas, tendencia y moldes. Este portal es pionero en la red nacional de internet brasileña, ya que todos los cortos disponibles, son proyectados en su formato original, sin cortes y respetando los derechos de autores de los realizadores. Portal Porta Curtas es patrocinado por Petrobrás.

Una de las características más llamativa del Portal Porta Curtas es que su objetivo reside en promocionar otros cortos a través de otras páginas web, lo cual garantiza una difusión mayor. Según las informaciones aportadas por el portal, "web masters, editores, blogueros pueden escoger aquellas películas que tengan un interés adicional, relacionadas con sus propias páginas webs, recibiendo un enlace el cual permite su producción. La provisión de estos enlaces en otras páginas web, es un servicio automático y totalmente gratuito al alcance de todo usuario" (Portal Porta Curtas, 2014). Por lo tanto, la circulación del contenido audiovisual no es limitada al portal.

\section{Proyecto Cidade Vellha}

El Proyecto Cidade Velha forma parte de un plano de cooperación transdisciplinário y corresponde a la creación de un museo virtual el cual ofrece una dimensión de la riqueza histórica y cultural la Ciudad Velha, situada en la Isla de Santiago, en Cabo Verde. Reconocida por la UNESCO como patrimonio mundial de la humanidad, Cidade Velha no se conoce solo por su turismo si no por el gran valor histórico que posee como ciudad. 
Las informaciones y datos del pasado de Cidade Velha eran bastante dispersas, por lo cual fue necesario encontrar una fuente para poder recopilar la información encontrada. Por eso, se diseñó el museo virtual Cidade Velha, como solución para poder facilitar la construcción de una base digital, la cual sea capaz de utilizar los beneficios que ofrecen las tecnologías de información y comunicación.

\section{Heurísticas de usabilidad y sociabilidad aplicadas a la contribución y colaboración: evaluación anallítica}

A partir de la evaluación analítica llevada a cabo por los expertos (Lobo, Barbosa, Ferreira, Ramos, Santos, 2015), se estructuraron cuatro tablas a partir de las heurísticas de usabilidad y sociabilidad, destacando el potencial de dichas plataformas.

\begin{tabular}{|c|c|c|c|}
\hline Usabilidad - Contribución & $\begin{array}{l}\text { Museu da } \\
\text { Pessoa }\end{array}$ & Porta Curtas & $\begin{array}{l}\text { Projecto Cidade } \\
\text { Velha }\end{array}$ \\
\hline $\begin{array}{l}\text { Low threshold interfaces for easily } \\
\text { making small contributions, e.g., no } \\
\text { login }\end{array}$ & - & - & - \\
\hline $\begin{array}{l}\text { High ceiling interdaces that allow large } \\
\text { and frequent contributions }\end{array}$ & $\checkmark$ & $\checkmark$ & $\checkmark$ \\
\hline $\begin{array}{l}\text { Visibility for users' contributions and } \\
\text { frequency of views; aggregated over } \\
\text { time }\end{array}$ & $\checkmark$ & $\checkmark$ & $\checkmark$ \\
\hline $\begin{array}{l}\text { Visibility of ratings and comments by } \\
\text { community members }\end{array}$ & $\checkmark$ & $\checkmark$ & $\checkmark$ \\
\hline $\begin{array}{l}\text { Tools to undo vandalism, limit } \\
\text { malicious sers, control pornography } \\
\text { and libel }\end{array}$ & $\begin{array}{l}\text { Registro, } \\
\text { email }\end{array}$ & $\begin{array}{l}\text { Registro, email, } \\
\text { comentarios }\end{array}$ & $\begin{array}{l}\text { Registro, email, } \\
\text { comentarios }\end{array}$ \\
\hline
\end{tabular}

Tabla III: Heurísticas de usabilidad para la contribución (Preece e Shneiderman, 2009).

\begin{tabular}{|lccc|}
\hline \multicolumn{1}{|c}{ Sociabilidad - Contribución } & $\begin{array}{c}\text { Museu da } \\
\text { Pessoa }\end{array}$ & $\begin{array}{c}\text { Porta Curtas } \\
\text { Support fro legitimate peripheral } \\
\text { participation so that readers can } \\
\text { gradually edge into contributing }\end{array}$ & $\begin{array}{c}\text { Projecto Cidade } \\
\text { Velha }\end{array}$ \\
\hline $\begin{array}{l}\text { A chance to build their reputation over } \\
\text { time while performing satisfying tasks }\end{array}$ & $\checkmark$ & $\checkmark$ & $\checkmark$ \\
\hline $\begin{array}{l}\text { Recognition for the highest quality and } \\
\text { quantity of contributions }\end{array}$ & $\checkmark$ & $\checkmark$ & $\checkmark$ \\
\hline $\begin{array}{l}\text { Recognition of a person's specific } \\
\text { expertise }\end{array}$ & $\checkmark$ & $\checkmark$ & $\checkmark$ \\
\hline $\begin{array}{l}\text { Policies and norms for appropriate } \\
\text { contributions }\end{array}$ & $\checkmark$ & $\checkmark$ & $\checkmark$ \\
\hline
\end{tabular}

Tabla IV: Heurísticas de sociabilidad para la contribución (Preece e Shneiderman, 2009). 


\begin{tabular}{|llll|}
\hline \multicolumn{1}{|c}{ Usabilidad - Collaboración } & \multicolumn{1}{c}{$\begin{array}{c}\text { Museu da } \\
\text { Pessoa }\end{array}$} & \multicolumn{1}{c}{ Porta Curtas } & $\begin{array}{c}\text { Projecto Cidade } \\
\text { Velha }\end{array}$ \\
\hline $\begin{array}{l}\text { Ways to locate relevant and competent } \\
\text { individuals to from collaborations }\end{array}$ & $\checkmark$ & $\checkmark$ & $\checkmark$ \\
\hline $\begin{array}{l}\text { Tools to collaborate: communicate within } \\
\text { groups, schedule projects, assign tasks, } \\
\text { share work products, request assistance }\end{array}$ & $\begin{array}{l}\text { Collección de } \\
\text { vídeos, } \\
\text { imágenes y } \\
\text { audio }\end{array}$ & $\begin{array}{l}\text { Cinemateca, } \\
\text { comentarios, } \\
\text { acervo } \\
\text { pedagógico }\end{array}$ & $\begin{array}{l}\text { Blog, wiki, galería de } \\
\text { vídeos e imágenes, } \\
\text { comentarios }\end{array}$ \\
\hline $\begin{array}{l}\text { Visible recognition and rewards for } \\
\text { collaborators e.g., authorship, citations, } \\
\text { links, acknowledgements }\end{array}$ & $\checkmark$ & $\checkmark$ & $\checkmark$ \\
\hline $\begin{array}{l}\text { Ways to resolve differences (e.g., voting), } \\
\text { mediate disputes, and deal with unhelpful } \\
\text { collaborators }\end{array}$ & Contactos & $\begin{array}{l}\text { Contactos, } \\
\text { comentarios, } \\
\text { votación }\end{array}$ & Contactos \\
\hline
\end{tabular}

Tabla V: Heurísticas de usabilidad para la colaboración (Preece e Shneiderman, 2009).

\begin{tabular}{|c|c|c|c|}
\hline Sociabilidad - Colaboración & $\begin{array}{l}\text { Museu da } \\
\text { Pessoa }\end{array}$ & Porta Curtas & $\begin{array}{l}\text { Projecto Cidade } \\
\text { Velha }\end{array}$ \\
\hline $\begin{array}{l}\text { An atmosphere to empathy and trust that } \\
\text { promotes belonging to the community } \\
\text { and willigness to work within groups to } \\
\text { produce something larger }\end{array}$ & $\checkmark$ & $\checkmark$ & $\checkmark$ \\
\hline $\begin{array}{l}\text { Altruism: a desire to support the } \\
\text { community, desire to give back, } \\
\text { willingness to reciprocate }\end{array}$ & $\checkmark$ & $\checkmark$ & $\checkmark$ \\
\hline $\begin{array}{l}\text { The desire to develop a reputation for } \\
\text { themselves and their collaborators, their } \\
\text { group and community; the need to } \\
\text { develop and maintain one's status within } \\
\text { the group }\end{array}$ & $\checkmark$ & $\checkmark$ & $\checkmark$ \\
\hline $\begin{array}{l}\text { Respect for one's status within the } \\
\text { community }\end{array}$ & $\checkmark$ & $\checkmark$ & $\checkmark$ \\
\hline
\end{tabular}

Tabla VI: Heurísticas de sociabilidad para la colaboración (Preece e Shneiderman, 2009).

Cuando comenzamos el análisis a partir de las heurísticas de usabilidad para la contribución (Tabla III) observamos que estas plataformas tienen la misma lógica para todas las heurísticas, con la excepción de la herramienta de comentario del Museo da Pessoa. En relación con las contribuciones de las interfaces, no hay necesidad de iniciar una sesión para acceder a ellas, ninguna posee este atributo. Para que el usuario pueda participar en las plataformas es necesario que este cree una cuenta, donde se informa del nombre, de la dirección email, creando una clave de acceso. De cierta forma, la elaboración de la cuenta del usuario, es considerada una herramienta fundamental, para así poder restringir el acceso a personas que tengan una mala intención y para así poder controlar la pornografía y difamación, como se señala la heurística de la tercera tabla.

Las tres plataformas permiten y facilitan la acción de contribuir, por ejemplo, el Museo da Pessoa, destaca en su página el enlace Participe, donde el usuario puede registrase como voluntario de la plataforma 
contribuyendo así en la transcripción y en la revisión de las historias de vida. Estas contribuciones se producen gracias a la promoción de dominio que hace la interfaz, como puede ser la seguridad y el apoyo que ofrece a los recién llegados. En cuanto a las bases del usuario, Porta Curtas es el único que posee FAQ, mientras las otras dos plataformas tienen sólo una sección de contactos.

La Tabla IV trata las heurísticas de sociabilidad para la contribución, las tres primeras son seguidas por las plataformas, ya que poseen herramientas de apoyo a la participación periférica (estos son los primeros pasos del usuario en la plataforma), también posibilitan construir una buena reputación al usuario, identificándolos gracias a los downloads realizados a través del Museo da Pessoa y en el Portal Porta Curtas.

Un ejemplo curioso en las primeras heurísticas es que en la plataforma del Museo da Pessoa no deja participar en sus herramientas, ya sean comentarios o clasificaciones de los recursos que ofrece (imagen, vídeo, texto y audio) la única opción que encontramos es "me gusta" y "compartir". Esta plataforma utiliza otros canales como pueden ser YouTube, Facebook, Google+, Instagram y Twitter, como repositorio de sus contenidos. Son solo en estos espacios que el usuario puede participar con los comentarios y clasificar los testimonios y noticias visualizadas.

Mientras que en la penúltima heurística de la Tabla IV, el reconocimiento de los atributos que el usuario gana, se realiza por otros participantes a través de los comentarios y clasificaciones. En el Portal Porta Curtas, todos los cortometrajes nacionales enviados, pueden ser valorados por los usuarios. Sobre este tema, encontramos algunos investigadores (Kuznetsov, 2006; Vassileva, 2003) los cuales afirman que dichas contribuciones por parte de otros participantes son importantes debido a la experiencia o a los valores de dichos participantes, pues son considerados un factor importante para la motivación. En el caso del Proyecto Cidade Velha, el reconocimiento por parte de estos usuarios es muy puntuales, debido a que este proyecto está en una fase experimental y no hay una dinámica en torno a las contribuciones publicadas.

Sobre la última heurística, las tres plataformas comparten políticas y normas de uso que están implícitamente colocadas en el archivo, en la descripción de los proyectos y en las diferentes etapas de contribución y colaboración del contenido. En el caso del Proyecto Cidade Velha, existe un administrador el cual modera las contribuciones. Si algo fuera abusivo o se encontrase fuera de contexto, es excluido.

Una vez finalizadas las heurísticas basadas en la etapa de contribución, pasamos a analizar la etapa colaborativa de las plataformas. Para comenzar tomamos por base las heurísticas presentadas en la Tabla $\mathrm{V}$, en que la primera trata de los diferentes mecanismos para atraer personas competentes y relevantes las cuales puedan actuar como colaboradores. Las tres plataformas hacen viable estos mecanismos, tanto por la propia temática colaborativa que posee, como por los iconos y enlaces que encamina al usuario al nivel de colaborador.

La segunda heurística hace referencia a las herramientas de usabilidad que son aplicadas al nivel colaborativo de las plataformas. Todas las plataformas poseen una herramienta en común, las galerías/colecciones/filmoteca de vídeo. El Museo da Pessoa se destaca por sus colecciones de audio, imagen y texto, las cuales son estructuradas por la colaboración de los usuarios. Ya que el Portal Porta Curta posee una base pedagógica en el en enlace Curta na Escola, que facilita la colaboración de profesores registrados, a 
través de sus experiencias en torno el uso de los cortometrajes nacionales en las aulas, comentarios de películas y experiencias educacionales, con historias/relatos que forman la Base de Datos de Relatos, la cual se encuentra abierta para consulta. En cuanto al Proyecto Cidade Velha, la plataforma también propone una colaboración por medio de la Wikipedia y del blog, con contenido relacionado en la historia y en la cultura de la Cidade Velha, en Cabo Verde.

A seguir, la tercera heurística habla sobre la visibilidad del reconocimiento y de la recompensa que se ofrece a los colaboradores de las plataformas. En todas ellas está reservada la autoría de los contenidos, los cuales tienen inicio al comenzar la sesión, así como citaciones y comparticiones de enlaces en otras redes con la preservación de contenidos colaborado.

Por fin, en la última heurística, la cual se corresponde a las herramientas para intermediar en conflictos y disputas, la cual se puede encontrar en todas las plataformas a través de la sección de contactos o por el propio email facilitado en el momento del registro. En el Portal Porta Curtas, esta mediación también se realiza con los comentarios y las votaciones que hay en torno a los contenidos.

Para finalizar este análisis, la Tabla VI trae las heurísticas de sociabilidad para la colaboración. La primera de ella, trata de una empatía y confianza promovidas por las plataformas, como una forma de garantizar la pertenencia del usuario como colaborador. De las tres plataformas, apenas el Proyecto Cidade Velha, y por ser un proyecto que se encuentra en una fase experimental, no sugiera esta pertenencia significativa de los colaboradores al proyecto.

Sobre las tres últimas heurísticas, todas ellas están unidas por una motivación de incentivar la contribución y colaboración en las plataformas: el altruismo (Maloney Krichmar e Preece, 2005; Vassileva, 2003). Con todo esto y con el altruismo, podemos ampliar esa reflexión para el concepto compromiso, el cual aparece adyacente, por surgir de la interacción del sujeto con el contexto (Finn \& Rock, 1997). Para Fredricks, Blumenfeld y Paris (2004), es posible enlazar tres tipos de compromiso: compromiso comportamental, el cual esta relacionado con la participación, el compromiso emocional, el cual relaciona las reacciones positivas y negativas del usuario, y el compromiso cognitivo, el cual hace referencia a la idea de investir.

Por medio de las estrategias de compromiso es que las plataformas proponen un acuerdo entre el usuario, la temática de colaboración y las plataformas. Por eso, es importante el planeamiento de las herramientas y de los recursos de las plataformas, ya que estos poseen una facilidad para envolver al usuario en un compromiso colectivo, especialmente cuando la propuesta es una concretización de las acciones colaborativas (Stahl, Koschmann \& Suthers, 2006). Así y a pesar de las heurísticas de sociabilidad en colaboración, destacaron el altruismo como motivador, al nivel de colaboración propuesto en las tres plataformas ya que se encuentra muy próximo al concepto compromiso.

De forma general, seria esencial poder resaltar que la comunicación con los usuarios, en todas las plataformas, se realiza de una forma asíncrona. La opción por este tipo de comunicación es muy ventajosa, y también por las herramientas que lo concretizan, pues el usuario puede contribuir/colaborar en su propio tiempo, y sus intervenciones se almacenan para que otras personas puedan ver, reaccionar y reconstruir el contenido (Naidu e Jarvela, 2006). 


\section{CONCLUSIONES}

El estudio analítico propuesto en este artículo tuvo como objetivo identificar el potencial y las restricciones en las plataformas multimedia relacionadas a la memoria cultural, en lo que se refiere a las heurísticas de usabilidad y sociabilidad para los niveles de contribución y colaboración. Partiendo de la idea que las heurísticas se apoyan como referencia en la literatura, Éstas contribuyen para la proyección de herramientas las cuales motiven la mejora del diseño de interacción y de la participación social de los usuarios mediados por la tecnología.

A partir de estas consideraciones que estructuramos aquí, se puede entender que alguna de las participaciones sociales on-line se trata de manera superflua. Alguna de ellas envuelven contribuciones regulares, otras sugieren una colaboración más estable, ya que la cultura de internet es mucho más que una transferencia de contenidos: se vuelve más social y comunal (Preece y Shneiderman, 2009). Por eso el incentivar a las personas a que contribuyan/colaboren resurge a través de los medios de acciones visibles, además del intercambio de capital social.

La confianza y la empatía son factores a tomar en cuenta, ya que desempeñan un papel singular en el sentido de incentivar a las personas a colaborar en el mundo on-line, así como ocurre en el offline (Preece, 1999; Maloney-Krichmar e Preece, 2005). El Museo da Pessoa es uno de los ejemplos, donde se observa con claridad la confianza que un contenido (especialmente los audiovisuales) puede producir que otros contenidos sean agregados, sobre todo para que futuros colaboradores vean la semejanza que hay entre ellos y las otras personas (Torrey et al., 2008), lo cual puede animar unos a otros a participar (Wu y Tshang, 2008). En este mismo nivel se encuentra el altruismo y el compromiso como factores de motivación para la contribución y colaboración.

Aunque se haya identificado el énfasis y el potencial de estas tres plataformas multimedia, otra cuestión a exponer seria la incoherencia de informaciones, la poca contribución del usuario en la propia plataforma y exceso de contribuciones en los repositorios. ¿Qué tipo de interfaz puede ser capaz de apoyar estos tipos de contribuciones y colaboraciones?

A pesar de que Preece y Shneiderman formulasen 16 heurísticas en torno a la contribución y colaboración, estos factores no fueron testados empíricamente, ya que para los propios autores existe una necesidad de desarrollar métricas para ambos procesos, como puede ser el numero de post, comentarios, fotos y vídeos, en los cuales sean posible medir el interés. Es un desafío que lanzamos y las plataformas que analizamos aquí pueden ser un punto de partida para así poder avanzar en la proyección de herramientas y recursos favoreciendo mas la parte social y comunal de la pagina web. 


\section{NOTAS}

Este término hace referencia a la Sociología de Émile Durkheim, donde las representaciones colectivas del mundo, incluyendo las del pasado, crean sus orígenes a partir de la interacción de los órganos sociales ya que no se puede reducir a simples contribuciones de los individuos.

El hombre crea y utiliza símbolos para asociar significado a todo. Y a través de estos símbolos se puede transmitir la cultura, de generación en generación, en una acción continuada de creación, transformación, mejora y recreación.

Estudiantes de doctorado vinculados en las investigaciones de información y comunicación en plataformas digitales, de la Universidad de Aveiro (Portugal).

\section{REFERENCIAS BIBLIOGRÁFICAS}

Barros, Ligia Alves (1994). Suporte a ambientes distribuídos para aprendizagem cooperativa. COOPE/Sistemas/UFRJ. Tese de Doutorado.

Butler, Brian; Joyce, Elisabeth; Pike, Jacqueline (2008). Don't look now, but we've created a bureaucracy: The nature and roles of policies and rules in Wikipedia. // Proceedings of 26th Annual ACM Conference on Human Factors in Computing Systems, 2008, 1101-1110. http://dl.acm.org/citation.cfm?id=1357227 (2015-01-10).

Carneiro, Henrique Figueiredo (2006). Banalização do patrimônio cultural e consequências perversas para a vida na cidade. // Martins, C. (org.). Patrimônio Cultural: da memória ao sentido do lugar. São Paulo: Roca.

Castells, Manuel (2001). A Era da Informação: Economia, Sociedade e Cultura, v. 3, São Paulo: Paz e Terra.

Chow, Anthony; Bridges, Michele; Commander, Patricia (2014). The Website Design and Usability of US Academic and Public Libraries. Reference \& User Services Quarterly 53(3), 2014, 253-266.

Convertino, Gregorio; Mentis, Helena M.; Rosson, Mary Beth; Carroll, John M.; Slavkovic, Aleksandra; Ganoe, Craig H. (2008). Articulating common ground in cooperative work: Content and process. // Proceedings of 26th Annual ACM Conference on Human Factors in Computing Systems, 2008, 1637-1646. http://dl.acm.org/citation.cfm?id=1357310 (201501-25).

Curta na Escola. http://www.curtanaescola.org.br/ (2015-01-10)

Denning, Peter J.; Yaholkovsky, Peter (2008). Getting to 'We': Solidarity, not software, generates collaboration. // Communications of the ACM (51) 4, 2008, 19-24.

Dix, Alan; Finlay, Janet; Abowd, Gregory D.; Beale, Russel (2004). Human-Computer Interaction. 3 ed. England: Pearson Education Limited. 
Duarte, Adriano (1999). Experiência, Modernindade e Campo dos Media. // Biblioteca On$\begin{array}{lllll}\text { line de } & \text { Ciências } & \text { da }\end{array}$ http://www.bocc.ubi.pt/ esp/autor.php?codautor=2 (2014-10-21).

Finn, Jeremy D.; Rock, Donald A. (1997). Academic success among students at risk for school failure. // Journal of Applied Psychology, 82, 1997, 221-234.

Fredricks, Jennifer A.; Blumenfeld, Phyllis C.; Paris, Alison H. (2004). School Engagement: Potential of the Concept, State of the Evidence. // Review of Educational Research, v. 74, n. 1, 2004, 59-109. http://rer.sagepub.com/content/74/1/59.short?rss=1\&ssource=mfc (2015-01-24).

Halbwachs, Maurice (2006). A memória coletiva. Trad. Beatriz Sidou. São Paulo: Centauro. Hilário, Ana B. Ríos (2014). Linked Open Media Data: La tecnología de datos vinculados al servicio de los medios de comunicación. // Cuadernos de Documentácion Multimédia. (25), 2014, ISNN:

1575-9733.

http://revistas.ucm.es/index.php/CDMU/article/view/47470/44478 (2015-02-24).

Kuznetsov, Stacey (2006). Motivations of contributors to Wikipedia. // ACM SIGCAS Computers and Society (36) 2, 2006, Article 1.

Lobo, Juliana (2013). Do argumento à cena: cinema em processo colaborativo no ciberespaço. Aveiro: Universidade de Aveiro, 2013, dissertação de Mestrado.

López-de-Quintana, Eugenio (2007). Transición y tendencias de la documentación en televisión: digitalización y nuevo mercado audiovisual. // El profesional de la información.16:5 (julio-agosto 2007) pp. 397-408. http://www.elprofesionaldelainformacion.com/contenidos/2007/septiembre/01.pdf (2015-04-24).

Maloney-Krichmar, Diane; Preece, Jenny (2005). A multilevel analysis of sociability, usability and community dynamics in an online health community. // ACM Transactions on Computer Human Interaction (12) 2, 2005, 1-32. http://dl.acm.org/citation.cfm?id=1067864 (2015-01-30).

Museu da Pessoa. http://www.museudapessoa.net/pt/home (2015-01-10).

Naidu, Som; Järvelä, Sanna. (2006). Analyzing CMC content for what?. // Computers \& Education, (46), 2006, 96-103. http://www.sciencedirect.com/science/article/pii/S0360131505000527 (2015-01-28). Nielsen, Jakob (2012). Usability 101: Introduction to Usability. 2012. http://www.nngroup.com/articles/usability101introduction-to-usability/ (2015-01-26). Portal Porta Curtas. http://portacurtas.org.br/ (2015-01-10).

Preece, Jenny. et al. (1994). Human-Computer Interaction. Reading. MA: Addison-Wesley. Preece, Jenny (2001). Sociability and usability in online communities: Determining and measuring success. // Behaviour \& Information Tech. 20, 5, 2001, 347-356. 
Preece, Jenny (1999). Empathic communities: Balancing emotional and factual communication. // Interacting with Computers, The Interdisciplinary Journal of HumanComputer Interaction (12) 1, 1999, 63-77.

Preece, Jenny; Shneiderman, Ben (2009). The Reader-to-Leader Framework: Motivating Technology-Mediated Social Participation. // AIS Transactions on Human-Computer Interaction, v. 1, issue 1, 2009, 13-32.

Projecto Cidade Velha. http://www.cidadevelha.gov.cv/ (2015-01-10).

Santaella, Lúcia; Nöth, Winfried (2005). Por que as comunicações e as artes estão convergindo?. São Paulo: Paulus.

Stahl, Gerry; Koschmann, Timothy; Suthers, Dan (2006). Computer-supported collaborative learning: An historical perspective. // R. K. Sawyer (Ed) Cambridge handbook of the learnin sciences. Cambridge, UK: Cambridge University Press, 2006. http://www.cis.drexel.edu/faculty/gerry/cscl/CSCL English.pdf. (2015-01-27).

Torrey, Cristen; Burk, Moira; Lee, Matthew; Dey, Anind; Fussell, Susan; Kiesler, Sara (2007). Connected Giving: Ordinary people coordinating disaster relief on the Internet. // Proceedings of 40th Annual Hawaii International Conference on System Sciences (HICSS). Vassileva, Julita (2003). Motivating participation in peer to peer communities. // P. Petta et al. (eds.) Engineering Societies in the Agents World III (ESAW 2002), Lecture Notes in Computer Science 2577, 2003, 141-155.

West, Joel; Gallagher, Scott (2004). Key challenges of open innovation: lessons from open source software. 2004. http://www.cob.sjsu.edu/west j/papers/WesrGallagher2004.pdf (2015-01-25).

Wu, Jhy-Jeng; Tsang, Alex S. L. (2008). Factors affecting members' trust belief and behaviour intention in virtual communities. // Behaviour and Information Technology (27) 2, 2008, 115-125. http://dl.acm.org/citation.cfm?id=1451123 (2015-01-24). 


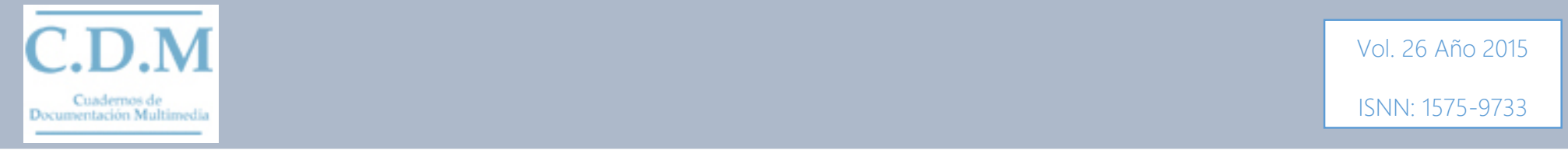

